\title{
Congenital Heart Disease in Adults. Considerations about Evolution, Natural and in Operated Patients
}

\author{
Edmar Atik, Fernando Antibas Atik \\ São Paulo, SP - Brazil
}

Thanks to diagnostic, therapeutic, and surgical advances, recent years have witnessed increasing numbers of congenital cardiopathic pediatric patients reach adulthood. Interest in this specialty among general cardiologists has followed, albeit with the obligatory aim of improving knowledge about the subject, albeit to learn about the most correct conduct to adopt in these cases. Such growing interest on the part of the cardiologist who treats adults is the consequence of the need to give the continuity required for adequate management of congenital cardiopathy starting most assuredly in early infancy.

To better discuss this subject, it is worth initially establishing guidelines for correct diagnosis and management. Congenital cardiopathy in the adult usually presents itself in two forms: that of natural evolution, and that of survivals of earlier surgery performed in breast-feeding newborns, infants, or during adolescence.

Natural evolution is of two kinds: one, with an unestablished previous diagnosis, showing discrete defects or even hemodynamic repercussions, and another, with a previously established diagnosis, but no surgical intervention having been performed due to counter-indication or lack of criteria indicating previous surgery.

The second form of presentation, postsurgical evolution reaching adulthood, includes patients who undergo one of three types of operations: palliative, correction of function, and anatomofunctional correction.

\section{Natural evolution, without previous diagnosis}

Discrete lesions - Cardiopathies of natural evolution, without previous diagnosis but with discrete hemodynamic repercussion defects, are passive to a non-surgical, expectant conduct in adults. They comprise a group of defects re-

Instituto do Coração do Hospital das Clínicas - FMUSP

Mailing address: Edmar Atik - InCor - Av. Dr. Enéas C. Aguiar 44 - 05403-000 São Paulo, SP - Brazil quiring clinical cognizance, in order to permit differentiation from resembling diseases and are represented by aortic arch anomalies without obstruction of blood flow and may occasionally simulate mediastinum tumors and inadvertently may even culminate in thoracotomy. Pertaining to this group are small atrial septal defect and partial anomalous drainage of the pulmonary veins. Even in the presence of discreteincrease in pulmonary blood flow, a differential diagnosis of arterial pulmonary hypertension should be avoided in view of the dilatation of the medial arch, as visualized by thoracic radiography. It should be mentioned that aortic coarctation, even when discrete, might become exteriorized by slight systemic arterial hypertension in the upper limbs or by more pronounced burst type hypertension.

A bicuspid aortic valve may be exteriorized by a systolic murmur with protosystolic clicking and occasionally even by a diastolic murmur of discrete aortic insufficiency; it should be distinguished from aortic valve stenosis.

A small ventricular septal defect should be recognized, because in case of acute myocardial infarction it may mistakenly be taken to have an ischemic cause and lead to unnecessary high-risk surgical procedures.

Corrected transposition of the great arteries with altered cardiac rhythm and with varying degrees of atrioventricular blockade, a distorted cardiac silhouette on thoracic radiography and an altered QS electrocardiographic morphology in V1 and V2 may raise diagnostic doubts about presumable ischemic cardiopathies.

Pronounced lesions - In contrast to the previously considered lesions, the pronounced lesions have a surgical indication in spite of a delayed diagnosis, possibly consequent to the greater tolerance of the patient to the overload imposed by the congenital defect. These lesions are mainly represented by atrial and ventricular septal defects, a partial defect of the atrioventricular septum, a patent arterial ductus, aortic coarctation and aortic stenosis. Among such cardiopathies, those with acquired adverse phenomena that do not regress even following correction, include presence of pulmonary arterial hypertension, systemic malignant arterial hypertension, secondary myocardiopathy, and 
intense ventricular dysfunction, as well as other vascular alterations.

\section{Natural evolution with previously known cardiopathy diagnoses}

Surgical counterindication - In cases where pulmonary hypertension is already in its evolutionary stage and regression does not occur even following adequate correction of defects surgery is not advised.

No actual indication for surgery - It is the conduct adopetd when the presumptive benefit to be obtained from an operation is minor, in discrete cardiopathies or having pronounced repercussions, in patients aged over 60 or 70 years where increased surgical risk is higher. Cardiopathies with systemic-pulmonary blood deviation, such as in atrial or ventricular septal defects, or a patent arterial ductus, most often fit into this framework, which also applies to obstructive cardiopathies.

This conduct extends to cardiopathies whose correction is rendered impossible by vascular lung alterations due to a decreased arterial tree insufficient for adequate pulmonary function, to the fragility of its walls or to other evolutionary alterations like in pulmonary atresia with ventricular septal defect and collateral systemic-pulmonary vessels.

\section{Postsurgical evolution}

Among the second group of anomalies experienced by adults is an important contingent related to patients who have survived previously performed operations.

In general, following palliative Blalock-Taussig type operations, complications related to cardiac volume overload with cardiac insufficiency prevail over time. Pulmonary artery dilatation and even pulmonary hypertension, as well as various degrees of hypoxemia dependent on the perviousness of systemic-pulmonary anastomosis may occur. A pulmonary artery cardiac insufficiency or hypoxemia banding also complicates with in this group.

Following functional surgery corrections like Fontan operation, systemic venous congestion, cardiac arrhythmia, cardiac failure, thrombosis, and pulmonary embolism can occur; more serious disturbances like altered enteric absorption and digestion problems are also found.

Senning and Mustard operations leading to supraventricular arrhythmia as well as to right cardiac failure and tricuspid insufficiency occur with a frequency of 20 to $50 \%$ in adult patients over an extended period of time.

In corrections made by interposition of tubes and valves between cardiac structures like the common arterial trunk, pulmonary atresia associated with ventricular septal defect, extreme tetralogy of Fallot, transpositions of great arteries with pulmonary stenosis and ventricular septal defect, a high incidence of obstruction and tissue degeneration with calcification, responsible for embolism and cardiac insufficiency are observed.
In corrective surgery, it is important to recognize the presence or absence of residual lesions, of sequelae and of complications that frequently influence long-term evolution of the patient. For example, obligatory pulmonary insufficiency following enlargement of the outlet of the right ventricle in tetralogy of Fallot, when pronounced, leads to right ventricle dilatation with signs of right cardiac insufficiency. In residual aortic coarctation, systemic arterial hypertension, aneurysms, secondary myocardiopathy, and coronariopathy may appear. Pulmonary stenosis leads to pulmonary insufficiency, aortic stenosis to aortic insufficiency, and so on.

In this context, whose outcome may or may not be unfavorable depending on the magnitude of residual defects, operated patients should be considered capable of attaining an anatomofunctional cure following, among acyanotic cardiopathies, atrial or ventricular septal defect or a patent arterial ductus, and among (previously feared) cyanotic anomalies, transposition of great arteries and anomalous total drainage of pulmonary veins, provided that surgery is performed very early in life and in the absence of an acquired irreversible phenomenon.

Experiencegained at the InCor Hospital-FMUSP-Sixhundred adult congenital cardiopathy patients aged between 19 and 68 years were treated; $333(55 \%)$ had natural evolution, and $267(45 \%)$ had postoperation evolution ${ }^{1}$. Of the first group, 203 patients $(60.9 \%)$ had clear-cut predominance of acyanotic cardiopathy with left-to-right blood deviation relative to $60(18 \%)$ acyanotic cardiopathies that had obstruction flow. Of 70 (21\%) cyanotic patients, $57(17.1 \%)$ had associated pulmonary stenosis and only 13 (3.9\%) had absence of pulmonary stenosis.

Of 203 shunted acyanotic cardiopathic patients, atrial septal defect in $135-(66.5 \%)$ patients predominated over ventricular septal defect in $30(14.7 \%)$ patients and atrioventricular septum defect in $27(13.3 \%)$ patients. Of 60 patients with obstructive defects, 26 (43.3\%) cases of pulmonary valve stenosis; predominated over 15 (25\%) cases of aortic coarctation, $10(16.6 \%)$ of aortic valve stenosis, and $9(115 \%)$ of corrected great artery transposition patients. Regarding cyanotic cardiopathies, tetralogy of Fallot occurring in 34-(48.5\%) and Ebstein anomaly occurring in 21 (30\%) patients were the most frequent anomalies; less common were double outlet from the right ventricle (5 patients, $7.1 \%$ ), pulmonary atresia with ventricular septal defect (4 patients, $5.7 \%$ ), great artery transposition (2 patients, $2.8 \%$ ), single ventricle (2 patients, $2.8 \%$ ), and tricuspid atresia $(2,2.8 \%)$. In the group of cardiopathies operated on in adults, 106 (54.9\%) acyanotic cardiopathies with shunting predominated over obstructive acyanotic cardiopathies (47, $24.3 \%$ ) and cyanotic cardiopathies (40, 20.7\%).

It is of interest that the majority of natural evolution cardiopathies had hemodynamic repercussion with the exception of $8(5.9 \%)$ of 135 patients with ventricular septal defect, $10(33.3 \%)$ of 30 patients with ventricular septal defect, 2 (18.1\%) of 11 patients with a patent arterial ductus, $8(30.7 \%)$ 
of 26 patients with pulmonary valve stenosis and 2 (22.2\%) of 9 patients with corrected great artery transposition, and 7 (33.3\%) of the 21 Ebstein anomaly patients.

Literature experience - Literature reports show that during natural evolution up to adulthood major cardiac defects have effects identical to those found in patients in our institution. Thus, among aortic coarctation patients, although the oldest survivor was 92 years old ${ }^{2}$, over $75 \%$ of the nonoperated patients died at around 50 years of age from systemic arterial hypertension-dependent cardiovascular alterations, like cerebral hemorrhage, aortic rupture or dissecting aneurysm, coronary obstruction, and secondary myocardiopathy with cardiac insufficiency ${ }^{3}$.

In pulmonary valve stenosis, the most common causa mortis was right ventricular insufficiency, usually appearing during the $4^{\text {th }}$ decade of life. Half of these patients die before reaching 27 years of age, but a long survival time of up to 78 years has been reported ${ }^{4}$.

Concerning atrial septal defect, the second most common congenital anomaly in adults, approximately $40 \%$ of patients survive beyond the age of 40 . One half die between the ages of 40 and 50 years; subsequent mortality occurs at a rate of $6 \%$ per year due to cardiac failure. However, survival periods of over 80 or even 90 years of age have been reported ${ }^{5}$.

The arterial ductus patient, a condition rarely found among adults, experiences cardiac insufficiency and infectious endocarditis, which are the most frequent causes of death in this age group along with arrhythmias like atrial fibrillation with ensuing thromboembolic phenomena.

Other complications like duct aneurysm, in particular when the arterial ductus is restrictive at the pulmonary anastosomotic mouth, are responsible for systemic embolism, laryngeal nerve paralysis, compression of the pulmonary trunk, and aneurysm rupture to the esophagus or the tracheo-bronchial tree. The oldest survivor with a natural evolution was 91 years old, with associated obstructive defects in the aortic and mitral valves ${ }^{6}$.

Ventricular septal defect is rarely found in adults, even though it is the second most frequent congenital anomaly. This is due to the spontaneous closure of around 50 to $75 \%$ of small defects during the first two years of life, as well as to the need for precocious operation of cases with a greater repercussion. The literature reports that spontaneous closure can occur even in adults, albeit rarely, as in cases of patients $23^{7}$ and $46^{8}$ years of age. Contradicting this assertion, others report spontaneous closure of the defect in a larger number of cases, including those of adults, attaining $10 \%$ of 188 patients aged between 17 and 72 years, averaging 29.2 years of age ${ }^{9}$.

During the evolution of a partial defect of the atrioventricular septum, the repercussion of mitral insufficiency adds to interatrial communication of the ostium primum type. For this reason, a survival of more than 50 years is rare and is estimated to occur in approximately $10 \%$ of the cases ${ }^{10}$.

The most common anomaly is still the bicuspid aortic valve, which in adults is exteriorized by aortic stenosis due to commisure fusion, calcic degeneration, or infectious endocarditis. The prevalence of this anomaly is estimated to reach $2 \%$ in a normal population.

Tetralogy of Fallot has an unfavorable precocious evolution, $50 \%$ of the patients dying during the first three years of life and $75 \%$ before reaching 10 years of age. About $11 \%$ of the cases reach the age of 20 years, $6 \%$ attain 30 years, and only $3 \%$ reach 40 years. In adults, aortic insufficiency due to valve ring and aortic dilatation and cardiac insufficiency consequent to chronic hypoxia are frequently observed.

In Ebstein anomaly, in contrast, half of the patients reach the $3^{\text {rd }}$ decade of life, but rarely reach 50 years of age $(5 \%)$. Death is due to cardiac failure, thromboembolism, arrhythmia, and hypoxia. Signs for a cautions prognosis are composed by exaggerated cardiomegaly, arrhythmia mainly of supraventricular paroxistic tachycardia and atrial fibrillation, cyanosis, and fatigue.

The highest survival rate has been reported in an 85 year-old patient ${ }^{11}$; it was first described, at the necropsy of a 75-year-old subject, who curiously despite extensive repercussion, had remained asymptomatic until reaching the age of 50 years ${ }^{12}$.

Natural occurrence of other cyanotic cardiopathies is extremely rare in adults, no predominance of one over the other having been observed.

Survival to adulthood following cardiac surgery-In general, long-term post-operation evolution depends on variables like the type of the congenital anomaly, age at the time of the intervention, degree of involution of the volume overload, the pressure made to bear on the ventricular myocardium, or both of these, myocardium protection during the operation, surgical sequels and complications, mainly of a physiological nature, and the durability of prosthetic materials like the valves and tubes used.

Aortic valve stenosis even when precociously corrected is an anomaly requiring attention in the adult in the face of progression of this defect, valve degeneration, appearance of aortic insufficiency, and of infectious endocarditis. Long-term survival times for this disease are estimated at $82 \%, 20$ years after the operation, and $77 \% 22$ years after the operation ${ }^{13}$. Need for reoperation increases with time, being $2 \%$ after 5 years, $8 \%$ after 10 years, $19 \%$ after 15 years, $35 \%$ after 20 years, and $44 \%$ after 22 years ${ }^{14}$. Sudden death is the main long-term causa mortis, occurring in $37 \%$ of all cases, $26 \%$ of which are related to the reoperation, $18 \%$ to endocarditis, and $4 \%$ to cardiac and aortic insufficiency ${ }^{14}$.

In subaortic stenosis, good results have been achieved on in the long-term following surgery, ever since technical changes have decreased the incidence of restenosis, as well as of complications like septal perforation, injuries to the bundle of His, the mitral valve, and also to the aortic valve ${ }^{15}$. Precocious indication for the repair of subvalve obstruction has avoided more pronounced alterations of the aortic valve, evoked by systolic jet impact. 
In aortic supravalve stenosis, mostly associated with Williams's syndrome, post-operative evolution remains related to previous damage to the aortic valve and the coronary circulation in the face of evoked preobstruction aortic dilatation and mechanical hypertension. Ninety-four percent favorable outcomes after 10 years and $91 \%$ after twenty years following operation, have been observed ${ }^{16}$.

Congenital mitral stenosis, an anomaly rarely appearing in its various forms of anatomical presentation, generally shows a more unfavorable evolution in cases where operative correction implies in obligatory stenotic lesions or even mitral valve insufficiency requiring future substitution by mechanical prostheses, accrued by the possibility of various grades of pulmonary hypertension and association with other cardiac defects. Survival times of 10 years following operation reach 30 to $60 \%$ in these cases ${ }^{17}$.

Long-term postoperation evolution of pulmonary valve stenosis is related to the intensity of the effect, age at operation and primarily mainly with previous functioning of the right ventricle. In general it is classified as excellent provided that the operation or even the intervention by a balloon catheter is precociously performed in the breast-feeding infants or during the first years of life ${ }^{18}$. In adults, the operation can lead to consequences when the right ventricle shows signs of dysfunction ${ }^{19}$.

In atrial septal defect, preoccupation with the need for precocious indication during the first years of life even in asymptomatic patients is centered on the fact that longterm involution of right cavity dilatation occurs, although when the operation is performed in adulthood, this only happens in $20 \%$ of the cases ${ }^{20}$.

Presence of tricuspid insufficiency and right ventricular dysfunction are, as a rule, continuous phenomena in patients operated on at an advanced age. Even so, correction is preferable to medical conduct, due to its better results both regarding quality of life and morbidity. During the follow-up of these operated patients (mean age, $54 \pm 10$ years), 10 year postoperation survival time were $95 \%$, but under medical treatment over the same period of time (mean age, $57 \pm 10$ years) survival corresponded to $84 \%$ of the cases ${ }^{21}$. Nevertheless, during postoperative evolution, supraventricular arrhythmias like atrial fibrillation or flutter and extrasystoles persist and may even arise, predisposing the patients to risks of thromboembolism.

Among the anatomical types of ventricular septal defect, those of in inlet portion and the perimembranous ones are usually corrected by the atrial route; subarterial types are corrected by right ventriculotomy, apical muscular ones by left ventriculotomy. For corrections performed precociously, generally prior to the age of two years, a long-term favorable evolution is observed, patients becoming asymptomatic with normal ventricular function in adult life $\mathrm{e}^{22,23}$.

When no involution of the cardiac dilatation imposed by a more prolonged overload prior to operation occurs, cardiomegaly and ventricular dysfunction may persist, and a greater possibility for the development of pulmonary hypertension is present ${ }^{24}$. It should be recalled that the time of survival of patients operated with an already pronounced pulmonary arteriolar reaction is in the order of $75 \%$ over 5 years ${ }^{25}$.

The evolution of the arterial ductus follows the same parameters established for ventricular septal defect, being related to age at operation, degree, and duration of the volume overload imposed on the left ventricle, and the degree of pulmonary hypertension.

Regarding aortic coarctation, the high incidence of recoarctation, the persistence of systemic arterial hypertension, the presence of frequently associated bicuspid aortic valve, and the formation of aortic aneurysm become constant sources of preoccupation after correction until adulthood. Delayed survival times are also influenced by frequent complications, like coronary sclerotic disease and cerebrovascular accidents. It becomes therefore, easy to imagine that longevity in this anomaly is influenced, clearly in an inverse relationship, by age at operation and the degree of acquired characteristics present, like arterial hypertension and myocardial hypertrophy ${ }^{26}$.

Therefore, when coarctation is corrected in infancy, longterm survival occurs in $92 \%$ of the cases; when correction is delayed (between 20 and 40 years of age), survival times decrease to $75 \%$ after approximately 25 years; $50 \%$ of patients over 40 years of age survive for 15 years following correction ${ }^{17}$.

Concerning arterial hypertension, patients become normotensive in $90 \%$ of the cases after an evolutionary period of 5 years if the correction is made after 5 years of age. This outcome however decreases to $50 \%$ of the cases after an evolutionary period of 20 years and to $25 \%$ after 25 years ${ }^{17}$. When the correction is performed in adulthood, normalization of arterial pressure is observed in only $20 \%$ of the cases, corresponding to a survival period of $67 \%$ for this age group ${ }^{27}$.

The bicuspid aortic valve constitutes another evolutionary risk, because it predisposes to commissure fusion and aortic valve stenosis as well as to infectious endocarditis.

Aortic re-coarctation, evaluated by the pressure gradient between members, above $20 \mathrm{mmHg}$ during rest and above $50 \mathrm{mmHg}$ during exercise, can occur during any corrective technique and constitutes a substrate for the consideration of a renewed surgical intervention or even balooncatheter angioplasty ${ }^{17,26,27}$.

Those with tetralogy of Fallot operated on during the first decade of their lives have an average survival rate of 32 years, corresponding to $90 \%$ compared with $76 \%$ of cases operated on during the same period at ages above 12 years ${ }^{28}$, operations in adulthood included ${ }^{29}$. The highest risk of the operation in adulthood is consequent to right ventricle hypertrophy, left ventricle volume overload imposed by previous palliative operations, and aided by cardiac rhythm disturbances.

The correction of tetralogy of Fallot is being performed by election ever more precociously during the first months and even the first month of life when the correction is simpler provided the distortion of the outlet from the right ventricle, and the ventricle hypertrophy are smaller, the arterial pulmonary tree tending to grow more rapidly in the postoperation period in face of the flow of ventricular contraction ${ }^{30}$.

In any situation, the major evolutionary factor besides 
the absence of residual defects like pulmonary valve stenosis and insufficiency and the residual ventricular septal defect proper is the systolic pressure relationship between the two ventricles when kept below $0.5^{30}$.

Pulmonary atresia associated with ventricular septal defect is a serious anomaly with such patients rarely reaching adulthood, with or without previous surgery. It has been estimated that around $16 \%$ of patients reach the third decade of life ${ }^{31}$ with a high incidence of anatomofunctional alterations, including aortic insufficiency in about $77 \%$, cardiac insufficiency in $38 \%$, with intense hypoxemia ${ }^{32}$. To this is added a high risk of arterial pulmonary hypertension.

In the transposition of great arteries, Jatene's anatomical correction propitiates significant anatomofunctional changes, which identify adult patients precociously operated on during the first year of life with preserved left ventricular function and the normal characteristics of this cavity regarding volume, pressure, and parietal thickness. The $2 \%$ risk of sudden death consequent to coronary disease following Jatene's correction, due to obstruction or around 10\% for pulmonary arterial obstruction, remain evolutional problems ${ }^{33}$. When the correction is aimed at redirecting intra-blood flow by means of the Senning and Mustard operations, a survival index of up to $85 \%$ by the first technique; and of $80 \%$ by the second has been reported after 20 years of evolution ${ }^{34}$.

Complications like vena cava stenosis with consequent syndrome of enteropathic loss of protein, pulmonary vein stenosis, tricuspid and right ventricle insufficiencies are becoming rarer over the years. Supraventricular arrhythmia remains a major cause of long-term cardiac decompensation ${ }^{34}$. Systemic right ventricular dysfunction arising out of inadequate coronary perfusion in the face of obligatory chronic hypertrophy also contributes as a primary cause of an unfavorable late evolution. However, the pathogenesis and incidence of this dysfunction still requires better studies by more sophisticated techniques such as myocardial metabolism using for example radioisotope perfusion.

On postoperation evolution, Ebstein's anomaly depends on the duration of tricuspid insufficiency and therefore on age at operation, functional condition of the right ventricle, residual lesions as well as the operation technique, performed by plastic surgery or valve substitution. A delayed mortality of up to $15 \%$ has been reported following correction of this anomaly by various techniques ${ }^{35,36}$.

\section{Reflections about long-term evolution of pa- tients after congenital heart disease surgery. Present panorama and required changes}

Late postoperation evolution of congenital cardiopathy carriers has raised innumerable doubts due to the variability of scientific information on the subject ${ }^{37,38}$. Such diversity depends on postoperation evolution of a series of variables requiring closer scrutiny; this in its turn, depends of the type of congenital cardiopathy involved, the anatomofuncional preoperation state of the patient, the type of the surgical correction whether anatomical or functional, eventual pre- and postoperation complications, as well as residual surgical defects. Along with these variables, the complexity of the subject, and the difficulty in understanding evolution analysis have to be considered. They presently serve as the basis for a better positioning in front of a congenital cardiopathy, establishing perspectives and consequently, alterations of medical conduct.

In a general way, present postoperation evolution of congenital cardiopathies can be of two major kinds: the first, considered favorable, in view of normal or close to normal population longevity and the second, considered unfavorable shows longevity shortened by one of the following possible factors: 1) presence of acquired adverse pre-operation factors becoming irreversible even following surgical correction; 2) existence of obligatory or nonobligatory postoperation residual defects; 3 ) functional and nonanatomical surgical corrections. Depending on their magnitude, these factors are responsible for decreased longevity over a greater or lesser period of time.

A favorable evolution is always related to cardiopathies correctable from the anatomical viewpoint; in general, precociously and in the absence of acquired adverse anatomical phenomena, with a preserved ventricular function and no complications or residual defects or both of these arising from the surgery.

Such anatomofunctional normality is obtained in acyanotic congenital cardiopathies with left to right deviation of blood, of the atrial and ventricular septal defect type, arterial ductus and even in atrioventricular septum defects and still, in arteriovenous fistulae, as in the origin of one of the pulmonary arteries from the ascending aorta provided that the anatomical corrections are precociously performed during the first months of life.

This favorable evolution is also extensive in acyanotic obstructive congenital cardiopathies (aortic and pulmonary stenosis and aortic coarctation) provided that the residual pressure gradient is persistently below $20 \mathrm{mmHg}$, and also to two cyanotic cardiopathies that today can be cured by corrective surgery and are represented by the transposal of the great arteries and by anomalous total drainage of the pulmonary veins.

Longevity is reduced by the presence of acquired adverse factors following cardiac hypertrophy and dilatation, along with pulmonary arterial hypertension, which are irreversible even in correctable congenital cardiopathy, performed late in adult life or during adolescence. This evolution occurs both during acyanotic obstructive congenital cardiopathies (pulmonary and aortic stenosis and aortic coarctation), as well as in cardiopathy with blood deviation from one to another circulation (atrial and ventricular septal defects and in the arterial ductus), provided that cardiac compensatory mechanisms overstep their limits following a given period of volume or pressure overload or both.

Following cardiopathy operated on at any age, residual surgery defects, be they obligatory or not, constitute factors that perpetuate overload, and markedly affect late evolution. For example, pulmonary insufficiency is labeled as obligatory following correction of tetralogy of Fallot, of 
causing volume overload of the right ventricle, proportional to the degree of the defect, bringing as a consequence, the appearance of right-sided cardiac insufficiency, arrhythmia, and low systemic output.

The residual right ventricular function in those with Ebstein anomaly, operated on as in adults in whom this cavity has undergone an exaggerated dilatation consequent to tricuspid insufficiency should also be brought out.

The insufficiencies of the right and left atrioventricular valves occurring to a greater or lesser extent following correction of the atrioventricular septum, in face of the magnitude of the malformation of the single atrioventricular valve, must also be mentioned among residual surgical defects. The residual defect under conditions of subvalve and even valve obstruction to the left and right sides of the heart is almost obligatory in face of malformations that render anatomical and therefore anatomofunctional normalization difficult. This assertion can be extended to isolated anomalies or to those associated with other defects like the transposition of great arteries, a double outlet from the right ventricle and the corrected transposition of the great arteries, also unfavorable for the evolution of cardiopathies whose correction is functional rather than anatomical, causing in the long run congestive, arrhythmic, embolic, and infectious complications. This evolution occurs in cardiopathies whose anatomical correction is not feasible, like tricuspid atresia, the atrioventricular-univentricular connection, left heart hypoplasia cardiopathy with hypoplasia of one of the ventricles, and certain congenital cardiopathies of difficult surgical correction. Fontan anastomosis, that is, systemic venous connection with the pulmonary arterial tree, is applicable in all of these conditions.

It may be added that functional correction is also applicable to situations in which the left ventricle loses the capacity to maintain systemic output, as in the transposition of the great arteries with an intact ventricular septum.

Functional correction in the transposition of the great arteries by intra-atrial redirecting of blood flow by the techniques of Mustard and Senning leads systemic venous blood to the left and pulmonary venous blood to the right ventricle, favoring systemic and pulmonary functional normalization. This effect is also applicable to other conditions like isolated atrioventricular discordance, in which the right atrium is connected to the left ventricle and the aorta, and the left atrium to the right ventricle and the pulmonary arteries. This functional correction is equally applicable in association with arterial and coronary exchange in corrected transposition of great arteries in the presence of pronounced tricuspid insufficiency.

Tube and valve placement according to the Rastelli principle, in the common arterial trunk, in pulmonary atresia with ventricular septal defect, in transposition of great arteries with pulmonary stenosis re-establishing right ventriclepulmonary trunk communication are part of the so-called functional corrections with all unfavorable consequences of degenerative, obstructive, and infectious phenomena.

It is clear that despite intense progress, perfection still looms far away. A gap in knowledge about etiological and pathogenetic aspects, as well as about genetic, biochemical, enzymatic and even prophylactic measures, which certainly would be most decisive steps toward the cure of congenital cardiopathies remains. To improve perspectives for this specialty, changes in conduct are a present-day necessity.

To avoid acquired adverse characteristics in correctable cardiopathies, it is imperative to perform precocious corrections during the first months of life. For noncorrectable cardiopathies, bearing in mind their usually unfavorable evolution, the solution for the now nearer future may lie in the use of more radical procedures like cardiac grafting.

Starting from a difficult beginning, we are presently still crossing a period of search for better horizons, to maintain the pledge for achievement of our main objective namely, to give cardiopathic children an evolution similar to that of healthy children.

\section{References}

1. Mesquita SF, Snitcowsky R, Ebaid M. Cardiopatia Congênita no Adulto. Em: Sousa AGMR, Mansur AJ: Socesp Cardiologia, $2^{\circ}$ vol. São Paulo: Editora Atheneu, 1996: 761-71.

2. Jarcho S. Coarctation of the aorta (Reynaud,1828). Am J Cardiol 1962; 9: 591-7.

3. Campbell M. Natural history of coarctation of the aorta. Br Heart J 1970; 32: 633-40.

4. Greene DG, Baldwin ED, Baldwin JS, Himmelstein A, Roh CE, Cournand A. Pure congenital pulmonary stenosis and idiopathic congenital dilatation of the pulmonary artery. Am J Med 1949; 6: 24-40.

5. Zaver AG, Nadas AS. Atrial septal defect-secundum type. Circulation 1965; 32(suppl III): 24-32.

6. Satoh T, Yanagitani Y, Okano Y. Patent ductus arteriosus with combined valvular disease at age 91. Intern Med 1997; 36: 340-4.

7. Schott GD. Documentation of spontaneous functional closure of a ventricular septal defect during adult life. Br Heart J 1973; 35: 1214-6.

8. Campbell M. Natural history of ventricular septal defect. Br Heart J 1971; 33: 246-57.

9. Neumayer U, Stone S, Somerville J. Small ventricular septal defects in adults. Eur Heart J 1998; 19: 1573-82.
10. Hynes JK, Tajik AJ, Seward JB, et al. Partial atrioventricular canal defect in adults. Circulation 1982; 66: 284-7.

11. Seward JB, Tajik AJ, Feist DJ, Smith HC. Ebstein's anomaly in an 85 year old man. Mayo Clin Proc 1979; 54: 193-6.

12. Adams JCL, Hudson R. Case of Ebstein's anomaly surviving to age 79. Br Heart J 1956; 18: 129-32.

13. Hsiesh K, Keane JF, Nadas AS, Bernhard WF, Castaneda AR. Long term follow-up of valvulotomy before 1968 for congenital aortic stenosis. Am J Cardiol 1986; 58: $338-41$

14. Presbitero P, Sommerville J, Revel-Chion R, Ross D. Open aortic valvulotomy for congenital aortic stenotic: late results. Br Heart J 1982; 47: 26-34.

15. Sommerville J, Stone $S$, Ross D. Fate of patients with fixed subaortic stenosis after surgical removal. Br Heart J 1980; 43: 629-47.

16. van Son JAM, Danielson GK, Puga FJ, et al. Supravalvar aortic stenosis: Long term results of surgical treatment. J Thorac Cardiovasc Surg 1994; 107: 103-14.

17. Kirklin JW, Barratt-Boyes BG. Cardiac Surgery, $2^{\text {nd }}$ ed. New York: Churchill Livingstone, Inc., 1993: 1263-325.

18. Kopecky SL, Gersch BJ, McGoon MD, et al. Long term outcome of patients 
undergoing surgical repair of isolated pulmonary valve stenosis: follow-up at 20 to 30 years. Circulation 1988; 78: 1150-6.

19. Perloff JK. Late postoperative concerns in adults with congenital heart disease. Cardiovasc Clin 1981; 11: 431-47.

20. Liberthson RR, Boucher CA, Strauss HW, Dinsmore RE, McKusick RA, Pohost GM. Right ventricular function in adult atrial septal defect. Am J Cardiol 1981; 47: 56-60.

21. Konstantinides S, Geibel A, Olschewski M, et al. A comparison of surgical and medical therapy for atrial septal defects in adults. NEngl J Med 1995; 333: 469-73.

22. Moller JH, Patton C, Varco RL, Lillehei CW. Late results (30 to 35 years) after operative closure of isolated ventricular septal defect from 1954 to 1960 . Am J Cardiol 1991; 68: 1491-7.

23. MeijboomF, Szatmari A, Utens E, et al. Long-term follow-up after surgical closure of ventricular septal defect in infancy and childhood. J Am Coll Cardiol 1994; 24: 1358-64.

24. Jarmakani JM, Graham TP, Canent RV. Left ventricular contractile state in children with successfully corrected ventricular septal defect. Circulation 1972; 45(suppl I): 102-10.

25. Blackstone EH, Kirklin JW, Bradley EL, DuShane JW, Applebaum A. Optimal age and results of repair of large ventricular septal defects. J Thorac Cardiovasc Surg 1976; 72: 661-79.

26. Presbitero P, Demarie D, Villani M, et al. Long-term results ( 15 to 30 years) of surgical repair of aortic coartation. Br Heart J 1987; 57: 462-7.

27. Clarkson PM, Nicholson MR, Barratt-Boyes BG, Neutze JM, Whitlock RM. Results after repair of coartation of the aorta beyond infancy: a 10 to 28 year follow-up with particular reference to late systemic hypertension. Am J Cardiol 1983; 51: 1481-8.

28. Murphy JG, Gersh BJ, Mair DD. Long-term outcome in patients undergoing surgical repair of tetralogy of Fallot. N Engl J Med 1993; 329: 593-9.
29. Hu DCK, Seward JB, Puga FJ, Fuster V, Tajik AJ. Total correction of tetralogy of Fallot at age 40 years or older: long-term follow-up. J Am Coll Cardiol 1985; 5: 40-4.

30. Walsh EP, Rockenmacher S, Keane JF, Hougen TJ, Lock JE, Castaneda AR. Late results in patients with tetralogy of Fallot repaired during infancy. Circulation 1988; 77: 1062-7.

31. Bull K, Somerville J, Ty E, Spiegelhalter D. Presentation and attrition in complex pulmonary atresia. J Am Coll Cardiol 1995; 25: 491-9.

32. Marelli AJ, Perloff JK, Child JC, Lacks H. Pulmonary atresia with ventricular septal defect in adults. Circulation 1994; 89: 243-51.

33. Kirklin JW, Blackstone EH, Tchervenkov CI, Castaneda AR and the Congenital Heart Surgeons Society: Clinical outcomes after the arterial switch operation for transposition: patient, support, procedural and institutional risk factors. Circulation 1992; 86: 1501-15.

34. Williams WG, Trusler GA, Kirklin JW, et al. Early and late results of a protocol for simple transposition leading to an atrial switch (Mustard) repair. J Thorac Cardiovasc Surg 1988; 95: 717-26.

35. Barbero-Marcial M, Verginelli G, Awad M, Ferreira S, Ebaid M, Zerbini EJ. Surgical treatment of Ebstein's anomaly: early and late results in 20 patients subjected to valve replacement. J Thorac Cardiovasc Surg 1979; 78: 416-22.

36. Westaby S, Karp RB, Kirklin JW, Waldon AL, Blackstone EH. Surgical treatment in Ebstein's malformation. Ann Thorac Surg 1982; 34: 388-95.

37. Perloff JK. Survival patterns without cardiac surgery or interventional catheterization. In: Perloff JK, Child JS. Congenital Heart Disease in Adults, $2^{\text {nd }}$ ed. Philadelphia: WB Saunders Co, 1998: 15-53.

38. Kaplan S, Perloff JK. Survival patterns after cardiac surgery or interventional catheterization. In: Perloff JK, Child JS. Congenital Heart Disease in Adults, $2^{\text {nd }}$ ed. Philadelphia: WB Saunders Co., 1998: 54-87. 